\title{
Forecasting Electric Power Generation of Grid-Connected Solar Photovoltaic Systems by Using Artificial Neural Networks
}

\author{
Wellington Maycon S. Bernardes* e F. M. de Vasconcelos** \\ *Universidade Federal de Uberlândia, Faculdade de Engenharia Elétrica, CEP 38.400-902, \\ Brasil (Tel: (34) 3239-4731; e-mail: wmsbernardes@ufu.br) \\ **Instituto Federal de Educação, Ciência e Tecnologia de São Paulo, CEP 01109-010, Brasil (e-mail: \\ vasconcelos.fillipe@gmail.com)
}

\begin{abstract}
The adoption of photovoltaic systems installed in homes and small/medium commercial and industrial buildings has grown considerably in the last decades in Brazil and worldwide. These systems, which are typically linked to primary (medium-voltage) or secondary (low-voltage) distribution networks, are called grid-connected power photovoltaic (PV) systems. Evaluating the return on investment of these systems requires a robust modelling and tools to prognosticate the amount of generated energy. This work, therefore, is based on the application of Artificial Neural Networks (ANN) to predict the daily-produced energy from a PV system installed at the University of São Paulo (USP). Meteorological and performance data were collected from this PV system for one month. This methodology may allow carrying out the efficiency analysis of the device, to plan the system operation and commercialization of the energy obtained strategically. We present several graphs which estimate the quality of the PV system, along with performance metrics, such as the number of epochs, mean squared errors and variances achieved in the simulations. Finally, the validation studies were performed by comparing the error between the experimentally measured data and the estimated data from ANN. The computational tests show that the purpose is interesting to solve the problem within an acceptable percentage error.
\end{abstract}

Resumo: A adoção de sistemas fotovoltaicos instalados em casas e pequenas e médias instalações comerciais e industriais tem crescido consideravelmente nas últimas décadas no Brasil e também internacionalmente. Esses sistemas, que são tipicamente ligados a redes primária (média tensão) ou secundária (baixa tensão) de redes de distribuição, são também chamados de sistemas fotovoltaicos de energia conectados à rede elétrica (SFCR's). Avaliar o retorno de investimento desses sistemas requerem um modelo robusto e ferramentas para diagnosticar a quantidade de energia gerada. Esse trabalho, portanto, baseado na aplicação de Redes Neurais Artificiais (RNAs) prediz a energia gerada no dia de um SFCR instalado na Universidade de São Paulo. Dados de desempenho e meteorológico foram coletados durante um mês. Essa metodologia pode permitir analisar a eficiência desse dispositivo, planejar a operação e comercialização da energia obtida estrategicamente. Diversos gráficos são apresentados que estimam a qualidade do SFCR, junto com métricas de desempenho, tais como número de épocas, erros quadráticos médios e variâncias obtidas nas simulações. Finalmente, os estudos de validação foram executados comparando os erros entre os dados medidos experimentalmente e os estimados pela RNA. Os testes computacionais mostram que o propósito é interessante para resolver o problema dentro de um erro percentual aceitável.

Keywords: Photovoltaic Systems; Artificial Neural Network; Power Forecasting; Distribution Systems; Artificial Intelligence.

Palavras-chaves: Sistemas fotovoltaicos; Redes Neurais Artificiais; Previsão de Energia Elétrica; Sistemas de Distribuição; Inteligência Artificial.

\section{INTRODUÇÃO}

As aplicações de Sistemas Fotovoltaicos (SFV's) estão amadurecendo cada vez mais, incentivadas pela necessidade e pelo interesse das nações em utilizar fontes renováveis de energia em casas, comércios e indústrias como uma forma de combate ao aquecimento global e à diversificação da matriz energética. Isso estimula a produção industrial em larga escala, além de incentivar pesquisas para redução de custos de componentes e aumento de desempenho destes sistemas. Medidas foram tomadas por governos e outras agências em alguns países, como Alemanha e Espanha, para subsidiar o custo de instalação e regulação a respeito da compra da eletricidade produzida por Sistemas Fotovoltaicos Conectados à Rede Elétrica (SFCR's), promovendo a disseminação do uso da energia solar para a geração de energia elétrica (Filho et al., 2010). No Brasil esse panorama também não é diferente: onde políticas públicas e estratégias 
estão gradativamente sendo implantadas para estimular este setor pouco explorado, sendo estas fundamentais para garantir a segurança energética do país (Júnior et al., 2017). A execução de um planejamento estratégico que aborde potenciais cenários se torna indispensável para minimizar despesas e viabilizar a operação dessa geração distribuída (Oliveira e Maria, 2017). Em um cenário de 5\% de penetração, a geração distribuída fotovoltaica acarreta em uma economia acumulada de R $\$$ 137,9 bilhões em saúde pública e remediação de danos ambientais. Além disso, ocorre o alívio na operação do SIN (Betz, 2019).

Normalmente, os SFCR's estão conectados às redes de distribuição primária ou secundária. A modelagem de sistemas fotovoltaicos é comumente utilizada na indústria para previsão do montante de energia gerado por SFCR's, dado o conjunto de condições climáticas (temperatura ambiente e irradiância). A modelagem de SFV's, todavia, tem uma vasta variedade de finalidades, dentre elas: monitoramento do desempenho do sistema; comparações dos diversos componentes de um SFV sem necessidade de comprá-los; cálculo de retorno de investimento (payback); etc. Modelos tradicionais para caracterizar os sistemas consistem em tratar o problema de maneira analítica para cada componente do sistema. O modelo do sistema é formado pela associação sucessiva de cada componente do SFV.

Diversos trabalhos na literatura abordam o tema sobre previsão de geração de energia em SFCR's (Raza et al., 2016). Mohammed e Aung (2016) usam um conjunto de diferentes algoritmos de aprendizagem de máquina, como árvore de decisão e classificador dos $K$ vizinhos mais próximos, para gerar previsões probabilísticas. Já Persson et al. (2017) aplicam árvores de regressão com Gradient Boosting em um horizonte de uma a seis horas. Relevantes variáveis meteorológicas e dados históricos de geração são usados para treinar o modelo. O Filtro de Kalman é usado em Takeda (2017) para predizer a geração elétrica usando dados mensais. A estimação de parâmetros do SFCR é também demonstrada. Por fim, Golestaneh et al. (2016) propõem uma aproximação não paramétrica para gerar densidades preditivas em curto tempo (até uma hora à frente).

Sabendo que a geração deve ser igual à carga em todo tempo e que qualquer fonte solar apresenta intermitência, é importante predizer essa geração renovável e alocar recursos para mitigar desbalanços. A predição, quando incorreta, pode se tornar substancialmente onerosa para o operador, seja por utilizar outras fontes mais caras disponíveis no momento, seja pela manutenção da geração renovável ociosa (Fatemi et al., 2016). Além disso, a avaliação do retorno de investimento desses sistemas requer que ferramentas de modelagem e de previsão do montante de energia gerado sejam utilizadas.

Este trabalho, por sua vez, tem a finalidade de propor uma RNA para caracterizar e modelar um SFV utilizado numa determinada instalação elétrica. Uma das grandes vantagens associadas à utilização de RNA, no que se refere à modelagem, é a praticidade. Esta técnica exige, como dados de entrada, apenas medições de algumas variáveis relevantes que se tornarão suficientes para a determinação de um modelo.
Destaca-se que as RNA são aceitas cientificamente na área de Sistemas Elétricos de Potência para diversas aplicações: estimação da velocidade de máquinas elétricas (Goedtel et al., 2013), estabilidade transitória (Abdallah et al., 2006) e classificação de cargas não lineares (Saraiva et al., 2015).

Aprofundando aqui, a relação entrada/saída "ensinada" à RNA adotada neste trabalho é a leitura de dados meteorológicos - temperatura ambiente $\left({ }^{\circ} \mathrm{C}\right)$ e irradiância no plano do gerador $\mathrm{FV}\left(\mathrm{W} / \mathrm{m}^{2}\right)$ - sendo dados de desempenho do SFCR - potência CA $(\mathrm{kW})$ e montante de energia gerado $(\mathrm{kWh})$ - a saída produzida pela RNA. Assim, uma das vantagens associadas à utilização da metodologia proposta é que esta não requer que se conheça todos os componentes individuais envolvidos e seus parâmetros de datasheet, isto é, potência nominal e número de painéis fotovoltaicos, potência nominal e eficiência do(s) inversor(es) etc.

Tendo dito isto, caracterizar e modelar SFCR's utilizando a metodologia proposta para instalações fotovoltaicas já existentes é o recomendável, já que somente nestes casos os dados meteorológicos e de desempenho poderão estar disponíveis de modo que um horizonte possa ser traçado em relação à previsão da geração de energia elétrica para as próximas semanas, meses ou anos.

Ademais, o rápido crescimento da indústria nos últimos anos expandiu o interesse e a necessidade da educação e treinamento ao nível mundial quando se trata de SFV. Neste sentido, este trabalho visa contribuir com o aprimoramento do estudo em SFCR's para a comunidade acadêmica e profissional do ramo na medida em que apresenta e avalia uma técnica para previsão da geração de energia elétrica por estes tipos de sistemas.

Este trabalho mostra particularidades de SFCR's assim, como as funcionalidades da técnica computacional desenvolvida. É importante comentar que existe um compromisso entre a precisão dos resultados e a simplicidade na implementação. No escopo, inclui-se também a concepção de avaliação de SFCR's e de previsão da geração visando a comercialização.

A validação experimental do programa foi realizada comparando suas previsões com os resultados realizados ao longo de um mês de funcionamento do SFCR integrado à edificação de um instituto da Universidade de São Paulo (USP), localizado na capital paulista. Todavia, destaca-se que este trabalho pode ser reproduzido em qualquer outra localidade sem perda de generalidade.

Este artigo é organizado no seguinte formato. A segunda seção oferece uma breve visão geral sobre SFCR's, elencando as variáveis envolvidas. A terceira seção delineia a metodologia adotada. A quarta seção explica as características do SFCR instalado na USP. Os resultados e suas análises são apresentados nas quinta e sexta seções, respectivamente. Por fim, as conclusões são apresentadas, bem como os apontamentos para futuras investigações.

\section{SISTEMAS FOTOVOLTAICOS CONECTADOS À REDE ELÉTRICA}

Na configuração básica de um SFCR, por simplicidade, consideram-se os conjuntos formados pelo gerador FV, o 
inversor e a rede elétrica local. O primeiro gera a energia em CC que será condicionada na unidade de potência e transformada em CA, para ser diretamente transferida à rede. Em cada etapa de processamento da eletricidade, há perdas relacionadas a diferentes causas, como, por exemplo, as perdas ôhmicas no cabeamento ou as perdas referentes ao processo de Seguimento do Ponto de Máxima Potência (SPMP). Essas perdas contabilizarão na eficiência da conversão da energia elétrica (Filho et al., 2010). Em adição, antes de abordar a modelagem dos SFCR's, é oportuno apresentar alguns parâmetros característicos que funcionam como indicadores de avaliação de desempenho de sistemas FV. Esses parâmetros característicos servem como ferramentas práticas importantes para a análise de SFCR's com base na produção de energia, no recurso solar e na potência instalada (Macêdo, 2006).

A Figura 1 mostra um diagrama esquemático da configuração básica do SFCR, bem como os parâmetros envolvidos na modelagem, detalhados a seguir (Zilles et al., 2012).

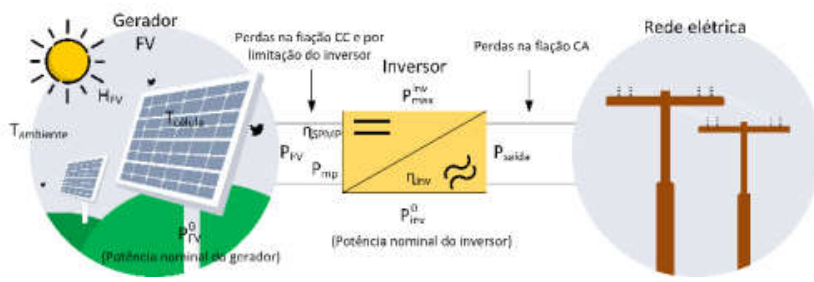

Fig. 1 Diagrama esquemático de um SFCR (Macêdo, 2006; Flaticon, 2017).

\subsection{Fator de capacidade (FC)}

É um parâmetro utilizado para estudo de desempenho de sistemas de produção de energia e varia muito entre as diferentes fontes de energia. Ele é definido como a razão entre a energia gerada em um determinado intervalo de tempo $\left(t_{2}-t_{1}\right)$ pela energia que poderia ser gerada se o sistema operasse todo o tempo na potência nominal, conforme a Eq. (1).

$$
F C=\frac{\int_{t 1}^{t 2} P_{\text {Saída }} \cdot d t}{P_{F V}^{0}\left(t_{2}-t_{1}\right)}
$$

onde: $P_{S a i ́ d a}$ é a potência instantânea gerada e $P_{F V}^{0}$ é a soma das potências nominais dos módulos FV que constituem o sistema.

\subsection{Produtividade do sistema $\left(Y_{F}\right)$}

$\mathrm{Na}$ área de sistemas $\mathrm{FV}$, o termo $Y_{F}$ é conhecido como produtividade do sistema, expressa em $\mathrm{kWh} / \mathrm{kWp}$, ou simplesmente em horas, que é a razão entre a energia entregue à rede elétrica em um determinado intervalo de tempo e a potência nominal do gerador, como mostra a Eq. (2).

$$
Y_{F}=\frac{\int_{t 1}^{t 2} P_{\text {Saída }} d t}{P_{F V}^{0}}
$$

Nota-se que o parâmetro $Y_{F}$ representa o número de horas que o sistema FV teria que operar com a potência nominal do gerador FV de modo a produzir a mesma quantidade de energia que foi entregue à rede elétrica (SFCR's) ou diretamente à carga (SFV autônomos). $\mathrm{O}$ parâmetro $Y_{F}$ normaliza a energia produzida levando em consideração o tamanho do sistema. Consequentemente, é uma maneira bastante conveniente para comparar energia produzida por sistemas fotovoltaicos de diferentes tamanhos, equipamentos, configurações etc.

Outro conceito importante é o $Y_{R}$ - ou Produtividade de Referência -, que é definido como a irradiação total no plano do gerador $\mathrm{FV}$, dividida pela irradiância de referência. A $Y_{R}$ corresponde ao número equivalente de horas de incidência da irradiância de referência $\left(1.000 \mathrm{~W} / \mathrm{m}^{2}\right)$ e é mostrada na Eq. (3).

$$
Y_{R}=\frac{\int_{t 1}^{t 2} H \cdot d t}{H_{r e f}}
$$

O $Y_{R}$ define a disponibilidade de recurso solar para um sistema FV, isto é, ela é função da localização, orientação do gerador FV e da variabilidade mensal e anual do clima.

\subsection{Rendimento global (Performance ratio)}

O Rendimento Global (PR) é definido como a razão entre $Y_{F}$ e $Y_{R}$, conforme a Equação (4). A partir dessa normalização com respeito à disponibilidade do recurso solar, são quantificadas as perdas em relação à potência nominal devido a diversos fatores, como: ineficiência do inversor, fiação, temperatura do módulo $\mathrm{FV}$, utilização incompleta da irradiância por reflexo na superfície frontal do módulo, sujeiras e falhas dos componentes.

$$
P R=\frac{Y_{F}}{Y_{R}}=\frac{\frac{\int_{t 1}^{t 2} P_{S a i ́ d a} d t}{P_{F V}^{0}}}{\frac{\int_{t 1}^{t 2} H \cdot d t}{H_{s, r e f}}}
$$

Os valores de PR normalmente são relatados em uma base mensal ou anual. Valores calculados para intervalos menores, tais como semanal ou diário, podem ser úteis para identificar ocorrências de falhas em componentes.

\section{METODOLOGIA}

A associação em série de células FV permite a construção de módulos FV que, por sua vez, quando conectados em uma matriz série-paralelo de módulos $\left(\mathrm{N}_{\mathrm{sG}} \times \mathrm{N}_{\mathrm{pG}}\right)$, constituem um gerador FV. A configuração série é essencial para atingir a tensão elétrica necessária e a configuração paralela é útil para alcançar a potência elétrica requerida. A parelela transforma a radiação solar em energia elétrica contínua em um processo regulado pela própria eficiência do gerador, sendo esta caracterizada por seu valor de potência nas denominadas condições padrão de medida (STC - Standard Test Conditions), e por um conjunto de fenômenos de segunda ordem relacionados com as condições de operação, sendo as principais: temperatura das células $\mathrm{FV}(\operatorname{costas})-T c\left({ }^{\circ} \mathrm{C}\right)-\mathrm{e}$ a incidência da luz solar perpendicular ao plano das células FV ou irradiância global no plano do gerador FV $-H\left(\mathrm{~W} / \mathrm{m}^{2}\right)$ (Martín, 1998). A literatura oferece diversos modelos que explicam adequadamente alguns destes fenômenos, 
entretanto, nestes modelos, em geral, as formulações matemáticas são bastante complexas (Gergaud et al., 2002).

Desta maneira, optou-se por uma modelagem baseada em uma rede neural artificial perceptron multicamadas (PMC), mais simples e de possível generalização, na qual, em um primeiro momento, a geração FV diária do SFCR estudado é estimada pela rede Time Delay Neural Network (TDNN). Em um segundo momento, uma rede PMC de Aproximação Funcional (AF) é avaliada, cujos dados de entrada são $T c$ e $H$, e a saída é a potência instantânea $-P_{G}(\mathrm{~W})$ - fornecida pelo gerador $\mathrm{FV}$.

A rede TDNN permite determinar a potência instantânea capaz de ser suprida por um gerador FV para todos os instantes de tempo definidos em um determinado dia com base em dados históricos de entrada e saída. Segundo este modelo, a saída estimada pela rede depende somente da $P_{G}$ medida nos dias anteriores. Já a rede $\mathrm{AF}$, por sua vez, determina $P_{G}$ somente se houver conhecimento de um conjunto de condições climáticas dado. Isto significa que, para este caso, a estimação da saída da rede depende do conhecimento de $T c$ e $H$ na condição atual em que a saída da rede é desejada.

Para realizar os estudos de previsão de geração de energia, portanto, realizaram-se medições de $T c, H$ e $P_{G}$, ponto a ponto, no intervalo de 5 minutos cada, para o mês de janeiro, no SFCR instalado na USP. Lembrando que o método descrito pode ser aplicado em qualquer período de tempo.

\section{DESCRIÇÃO DO SFCR INSTALADO NA USP}

$\mathrm{O}$ gerador FV é composto pela associação em paralelo de 2 conjuntos em série de 12 módulos fotovoltaicos cada, silício policristalino $(p c-S i)$, da fabricante SOLAREX, modelo MSX83, cuja potência nominal unitária é aproximadamente de 83 Wp. A Figura 2 ilustra a configuração da associação elétrica dos painéis e conexão com o inversor de frequência.

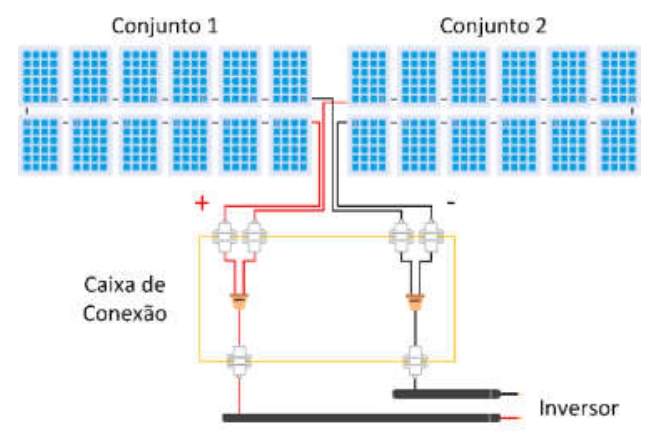

Fig. 2 Configuração da associação elétrica dos módulos fotovoltaicos (Macêdo, 2006; Flaticon, 2017).

O inversor usado é o SUNNY BOY modelo SB1100, de 1 $\mathrm{kW}$ de potência nominal, e trabalha numa ampla faixa de tensão CC de entrada (139 V - 320 V).

O Fator de Dimensionamento do Sistema (FDI), que corresponde à relação da potência nominal do inversor pela potência instalada do gerador $\mathrm{FV}$, e é calculada em FDI = 0,52 , assumindo que a potência instalada do grupo gerador é aproximadamente de $1.940 \mathrm{Wp}$.

\section{RESULTADOS COMPUTACIONAIS}

Simulações computacionais foram feitas no sistema descrito. 5 estudos de casos são apresentados para a rede TDNN e um para a rede AF. Para mais informações a respeito da implementação das redes TDNN e AF, consulte Silva et al. (2010). Em cada estudo de caso, quantidade de neurônios, quantidade de entradas, conjunto de treinamento e/ou conjunto de operação são distintos, cuja finalidade é contribuir para a verificação da aplicabilidade de uma TDNN e de uma AF para a previsão de geração de energia elétrica em SFCRs.

Os dados apresentados nesta seção estão todos normalizados com base nos valores máximos identificados no banco de dados. Este valor-base adotado para a normalização da saída $\mathrm{P}_{\mathrm{G}}$, portanto, foi de $1.099 \mathrm{~W}$. Os pesos sinápticos foram todos inicializados em valores compreendidos entre zero e um. A taxa de aprendizagem foi estabelecida com o valor de 0,001 .

A apresentação dos resultados em todos os estudos de caso está compreendida no intervalo das $6: 00 \mathrm{~h}$ às $18: 30 \mathrm{~h}$, pois esta pode ser uma boa aproximação para o período em que o sol está nascendo e se pondo, respectivamente. Assim, o conjunto de dados representativo de um dia completo é composto por 151 pontos ou instantes.

Os resultados e os detalhes de todos os estudos de caso usando cada uma das redes são apresentados a seguir, sendo os parâmetros atribuídos às redes definidos como: (a) número de neurônios da camada oculta $(\mathrm{N})$; (b) número de entradas ou ordem do preditor (X); (c) quantidade de amostras de treinamento (T); e (d) quantidade de amostras de validação (V).

\subsection{Rede $\operatorname{TDNN}(N=25, X=15, T=1510, V=151)$}

Este primeiro estudo de caso contempla a aplicação de uma rede TDNN com 25 neurônios na camada oculta e 1 na camada de saída, correspondente à $P_{G}$.

O conjunto de treinamento foi formado por 1510 pontos, o que equivale a 10 dias de medições, sendo estes extraídos dos dias 01 a 10 de janeiro. As energias totais geradas ao longo de cada dia são ilustradas na Figura 3.

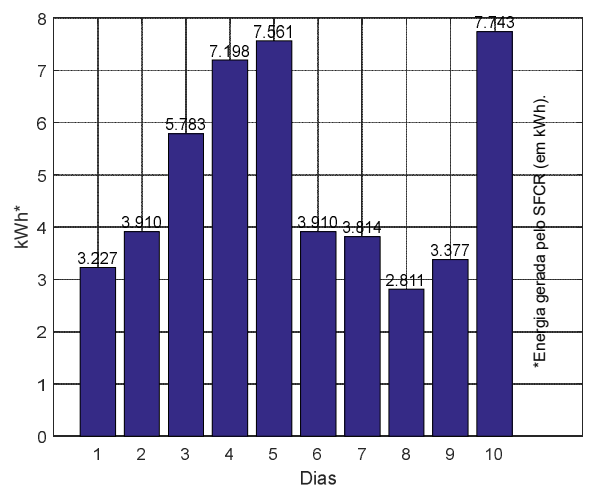

Fig. 3 Energias totais geradas ao longo de cada dia do SFCR instalado na USP para o mês de janeiro.

A partir dos resultados obtidos, vale ressaltar que é possível identificar que, no conjunto de treinamento, foram 
contemplados dias ensolarados, como os dias 4, 5 e 10, dias parcialmente nublados, como os dias 2, 3, 6 e 7, e dias chuvosos e/ou muito nublados, como os dias 1 e 8 .

O conjunto de dados de validação corresponde a 151 pontos, extraídos no dia 11 de janeiro, e a energia gerada pelo SFCR neste dia corresponde a $8,00542 \mathrm{kWh}$.

Os resultados obtidos quando foram confrontados os valores desejados e os estimados pela TDNN são ilustrados na Figura 4.

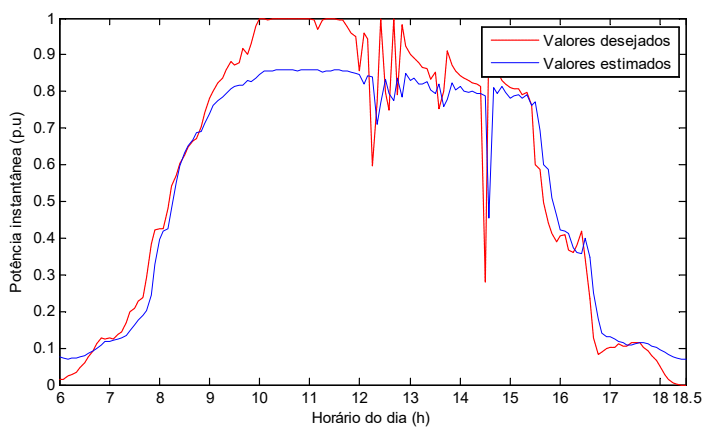

Fig. 4 Ilustração dos valores desejados e estimados pela TDNN para potência instantânea (em p.u) gerada pelo SFCR ao longo do dia 11 de janeiro. Estudo de caso 1.

Observa-se que o perfil da curva estimada é bastante semelhante em relação à desejada. Por outro lado, principalmente nos momentos de picos, identifica-se que a RNA empregada não foi capaz de determinar com alta precisão a saída desejada porque uma rede TDNN não foi capaz de prever - baseado somente em valores passados - os instantes em que o dia está bastante ensolarado, como pode ser visto nos horários compreendidos entre as 8:30 e 12:30 h. Isto pode ser entendido na medida em que o conjunto de treinamento contempla dias chuvosos, parcialmente nublados e ensolarados.

Alguns aspectos relevantes do treinamento e da validação destes resultados:

- Número de épocas até a convergência: 1996

- Erro Quadrático Médio (EQM): 0,0063

- Variância: 9,9290

\subsection{Rede $\operatorname{TDNN}(N=15, X=25, T=1510, V=151)$}

O segundo estudo de caso contempla a aplicação de uma rede TDNN para o mesmo caso $\mathrm{A}$, todavia, o número de neurônios na camada oculta $(\mathrm{N})$ é reduzido para 15 e a ordem do preditor (X) aumentada para 25.

$\mathrm{O}$ conjunto de treinamento é o mesmo apresentado anteriormente, sendo formado por 1510 pontos, o que equivale a 10 dias de medições, sendo estes extraídos dos dias 01 a 10 de janeiro.

O conjunto de dados de validação também é equivalente, sendo correspondente a 151 pontos extraídos do dia 11 de janeiro, cuja energia gerada foi de $8,00542 \mathrm{kWh}$.

Os resultados obtidos são ilustrados na Figura 5.

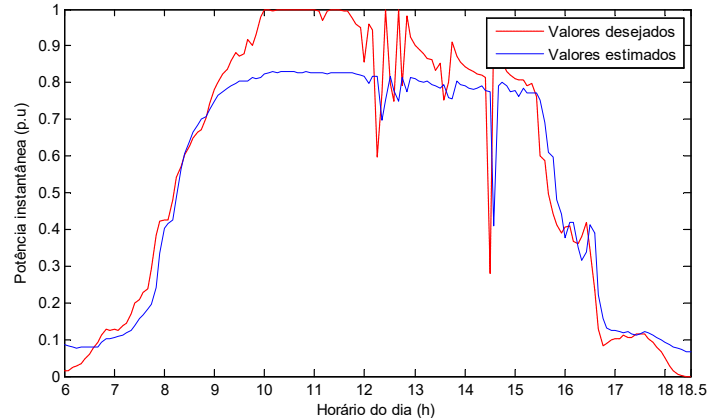

Fig. 5 Ilustração dos valores desejados e estimados pela TDNN para potência instantânea (em p.u) gerada pelo SFCR ao longo do dia 11 de janeiro. Estudo de caso 2.

Alguns aspectos relevantes do treinamento e da validação destes resultados:

- $\quad$ Número de épocas até a convergência: 1768

- $\quad$ Erro Quadrático Médio (EQM): 0,006356

- Variância: 9,5024

4.3 Rede $\operatorname{TDNN}(N=20, X=35, T=1510, V=151)$

O terceiro estudo de caso contempla a aplicação de uma rede TDNN cujo conjunto de treinamento contempla 10 dias de medições, dos dias 02 a 11 de janeiro, de modo que o conjunto de dados de validação corresponde a 151 pontos extraídos do dia 12 de janeiro. A energia gerada pelo SFCR neste dia foi de $5,73058 \mathrm{kWh}$.

Os resultados obtidos são ilustrados na Figura 6.

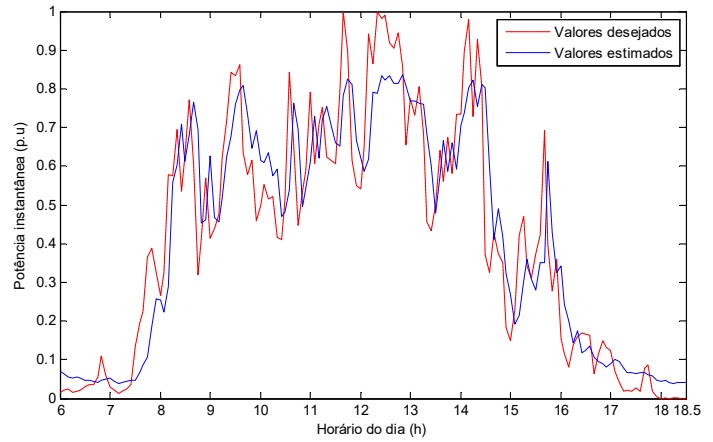

Fig. 6 Ilustração dos valores desejados e estimados pela TDNN para potência instantânea (em p.u) gerada pelo SFCR ao longo do dia 12 de janeiro. Estudo de caso 3.

Alguns resultados relevantes em relação ao treinamento e à validação:

- Número de épocas até a convergência: 1664

- Erro Quadrático Médio (EQM): 0,0063

- Variância: 8,4426

4.4 Rede $\operatorname{TDNN}(N=15, X=10, T=1510, V=151)$

O quarto estudo de caso contempla a aplicação de uma rede TDNN cujos conjuntos de treinamento e de validação contemplam os mesmos dados do estudo anterior, de modo que haja variação somente no número de neurônios da 
camada oculta $(\mathrm{N})$, de 20 para 15 , e na ordem do preditor $(\mathrm{X})$, de 35 para 10 .

Os resultados obtidos são ilustrados na Figura 7.

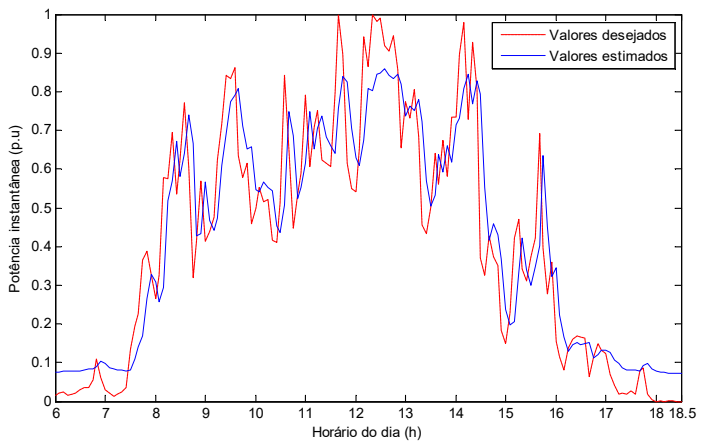

Fig. 7 Ilustração dos valores desejados e estimados pela TDNN para potência instantânea (em p.u) gerada pelo SFCR ao longo do dia 12 de janeiro. Estudo de caso 4.

Alguns resultados relevantes em relação ao treinamento e à validação:

- Número de épocas até a convergência: 1992

- Erro Quadrático Médio (EQM): 0,0064

- Variância: 7,6786

4.5 Rede $\operatorname{TDNN}(N=15, X=10, T=1510, V=151)$

Neste quinto estudo de caso, a aplicação da rede TDNN foi feita uma filtragem nos dados do conjunto de treinamento para estar mais adequado aos conjuntos de dados de validação.

O conjunto de treinamento foi formado por 1510 pontos, o que equivale a 10 dias de medições, sendo estes extraídos dos dias $04,05,10,11,13,14,17,18,19$ e 20 de janeiro. As energias totais geradas ao longo de cada dia são ilustradas na Figura 8.

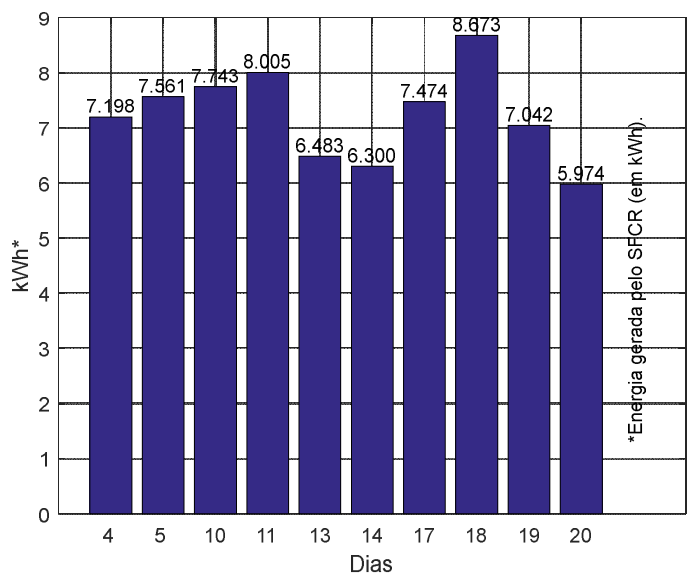

Fig. 8 Energias totais geradas ao longo de cada dia do SFCR instalado na USP para o conjunto de treinamento.

A partir dos resultados obtidos, vale ressaltar que é possível identificar que no conjunto de treinamento foram contemplados somente dias considerados como ensolarados ao longo do mês de janeiro.

Diante de um conhecimento prévio que o dia a ser estimado pela RNA será também ensolarado, tomou-se como conjunto de dados de validação 151 pontos extraídos do dia 16 de janeiro, sendo a energia gerada pelo SFCR neste dia correspondente a $6,42200 \mathrm{kWh}$.

Os resultados obtidos, quando foram confrontados os valores desejados e os estimados pela TDNN, são ilustrados na Figura 9.

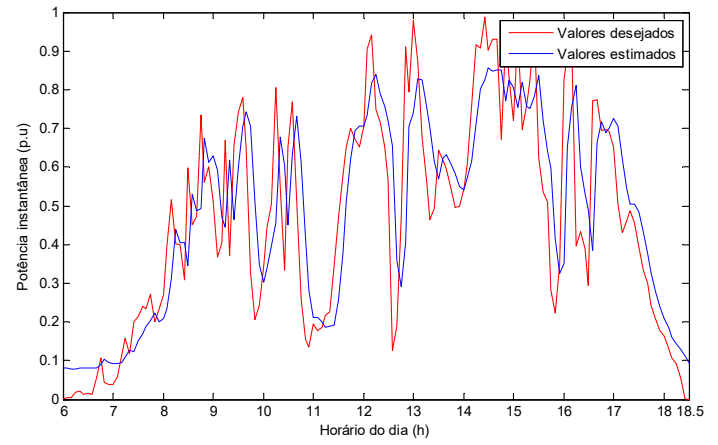

Fig. 9 Ilustração dos valores desejados e estimados pela TDNN para potência instantânea (em p.u) gerada pelo SFCR ao longo do dia 16 de janeiro. Estudo de caso 5.

Alguns resultados relevantes em relação ao treinamento e à validação:

- Número de épocas até a convergência: 1447

- Erro Quadrático Médio (EQM): 0,00866

- Variância: 6,18376

4.6 Rede $A F(N=25, X=151, T=1510, V=151)$

Este sexto estudo de caso contempla a aplicação de uma rede AF com 25 neurônios na camada oculta e 1 na camada de saída, correspondente à $P_{G}$.

O conjunto de treinamento e o conjunto de dados de validação são os mesmos do primeiro estudo de caso, sendo estes formados por 1510 pontos, o que equivale a 10 dias de medições, sendo estes extraídos dos dias 01 a 10 de janeiro para o treinamento, e 151 pontos extraídos do dia 11 de janeiro para validação.

Os resultados obtidos, quando foram confrontados os valores desejados e os estimados pela rede, são ilustrados na Figura 10.

Alguns resultados relevantes em relação ao treinamento e à validação:

- Número de épocas até a convergência: 94

- Erro Quadrático Médio (EQM): 0,0033

- Variância: 13,3284

\section{ANÁLISE DOS RESULTADOS}




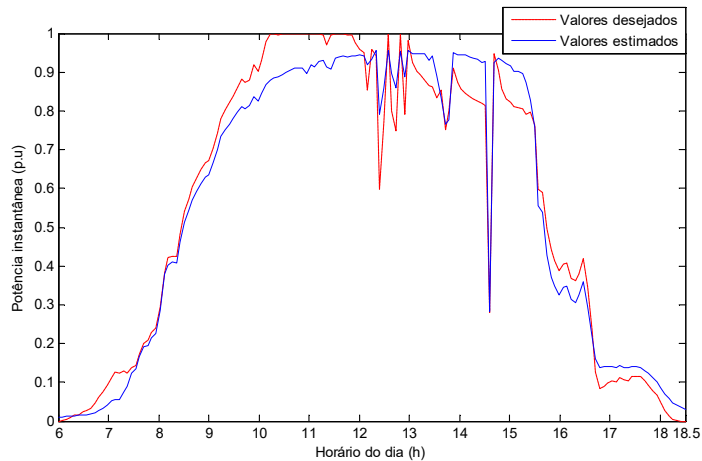

Fig. 10 Ilustração dos valores desejados e estimados pela rede AF para potência instantânea (em p.u) gerada pelo SFCR ao longo do dia 11 de janeiro. Estudo de caso 6.

As estimativas obtidas pela TDNN nos cinco estudos de casos foram confrontadas com os valores desejados. É possível observar nos resultados apresentados para todos os casos que a rede TDNN é capaz de traçar o perfil da curva de geração de energia elétrica por um SFCR com uma precisão bastante satisfatória, considerando o dinamismo encontrado nestes tipos de sistemas. Para permitir a avaliação dos resultados das redes implementadas, os valores dos parâmetros característicos (vide seção II) foram calculados usando as medições reais e os valores estimados, respectivamente, e são apresentados e confrontados nas Tabelas 1 e 2, calculando-se o respectivo erro.

Tabela 1. Parâmetros característicos do SFCR utilizando os valores medidos pelo sistema de aquisição de dados.

\begin{tabular}{|c|c|c|c|c|}
\hline SFCR & $\begin{array}{c}\boldsymbol{E}_{\text {GERADA }} \\
\mathbf{( k W h )}\end{array}$ & $\boldsymbol{F C} *$ & $\boldsymbol{Y}_{\boldsymbol{F}}(\mathbf{k W h} / \mathbf{k W p )}$ & $\boldsymbol{P R}^{*}$ \\
\hline Janeiro** & 159,52433 & 0,1105 & 82,229 & 0,6455 \\
$11 /$ jan & 8,00542 & 0,1719 & 4,1265 & 0,6055 \\
12 / jan & 5,73058 & 0,1231 & 2,9539 & 0,6587 \\
16 / jan & 6,42220 & 0,1379 & 3,3103 & 0,6747 \\
\hline
\end{tabular}

* Valores adimensionais.

**Dados do sistema sendo analisados para os 31 dias do mês de janeiro.

Tabela 2 - Parâmetros característicos do SFCR utilizando os valores estimados nos estudos de casos $\mathrm{e}$ erro percentual em relação aos valores desejados.

\begin{tabular}{|c|c|c|c|c|}
\hline SFCR & $\begin{array}{c}\mathrm{E}_{1} \\
(\mathrm{kWh})\end{array}$ & $F C_{I}^{*}$ & $\begin{array}{c}Y_{F I}(\mathrm{kWh} / \\
\mathrm{kWp})\end{array}$ & $P R_{I}^{*}$ \\
\hline TDNN A & 7,5921 & 0,1631 & 3,9135 & 0,5743 \\
\hline Erro $A^{* *}(\%)$ & 5,1432 & \multicolumn{3}{|c|}{5,16296} \\
\hline TDNN B & 7,4346 & 0,1597 & 3,8323 & 0,5623 \\
\hline Erro B** (\%) & 7,1109 & \multicolumn{3}{|c|}{7,13038} \\
\hline TDNN C & 5,7735 & 0,1240 & 2,9760 & 0,6637 \\
\hline Erro $C^{* *}(\%)$ & $\mathbf{0 , 8 0 0 5}$ & \multicolumn{3}{|c|}{0,74891} \\
\hline TDNN D & 5,8988 & 0,1267 & 3,0406 & 0,6781 \\
\hline Erro D** (\%) & 2,9874 & \multicolumn{3}{|c|}{2,93542} \\
\hline TDNN E & 6,6156 & 0,1421 & 3,4101 & 0,6951 \\
\hline Erro E** (\%) & 3,0153 & \multicolumn{3}{|c|}{3,01464} \\
\hline $\mathbf{A F}$ & 7,9054 & 0,1698 & 4,0749 & 0,5979 \\
\hline $\begin{array}{l}\text { Erro AF** } \\
(\%)\end{array}$ & 1,2282 & \multicolumn{3}{|c|}{1,24936} \\
\hline
\end{tabular}

$*$ Valores adimensionais.

** Valor absoluto dos valores desejados subtraídos dos estimados pela rede neural.

Das Tabelas 1 e 2, pode-se concluir que os erros foram relativamente elevados nos TDNN A e B, embora ainda satisfatórios, dependendo do nível de precisão desejado. Já os
TDNN C, D, E e AF, apresentaram boa precisão. Comparando os dados das Tabelas 1 e 2 quanto aos parâmetros característicos, sendo estas ferramentas importantes para a análise e caracterização de SFCRs, verifica-se boa concordância também entre os resultados, considerando que as TDNN A, B e a rede AF devem ser validadas com os dados de $11 /$ jan., as redes TDNN C e D, com 12 / jan., e, por fim, a rede TDNN E com $16 /$ jan. Neste cenário, alguns detalhes são analisados mais criteriosamente em cada estudo de caso e serão expostos a seguir.

Nos estudos de casos A e B, é de interesse atentar para o conjunto de treinamento utilizado neste tipo de problema. Em ambos foram utilizados os 10 primeiros dias do mês de janeiro, estando no período inclusos dias ensolarados, parcialmente nublados e chuvosos e/ou nublados, de modo que os dados de validação foram os pontos do dia 11 de janeiro. Um estudo de caso diferenciou-se do outro somente pelo número de neurônios em cada camada oculta e pela quantidade de dados de entrada da rede, o que - como pode ser visto na Tabela 2 - ocasionou diferenças, principalmente no cálculo da energia gerada ao longo do dia, sendo o erro da TDNN A igual a 5,14\% e da TDNN B equivalente a 7,11 $\%$. Uma característica que pôde ser evidenciada em ambas é que a TDNN teve dificuldades de acompanhar o perfil da curva de carga nos momentos mais ensolarados do dia. Uma interpretação plausível para isto é que se deve ao conjunto de treinamento, que incluía dados de dias nublados e chuvosos, fazendo com que a resposta da rede não atingisse amplitudes maiores.

Nos estudos de casos C e D, é de interesse atentar que os erros entre os valores desejados e estimados foram bastante pequenos, pois o conjunto de validação correspondia a um dia parcialmente nublado, valor relativamente intermediário quando comparado aos valores do conjunto de treinamento, que incluía dias ensolarados e chuvosos também.

No estudo de caso TDNN E, visando contornar o problema apresentado nos TDNN A e B, a rede foi treinada somente com dados de dias ensolarados e a validação foi realizada também para um dia em que se sabia que seria ensolarado. Os resultados dos valores estimados desta rede demostram melhora, pois, conforme Tabela 2, apresentou um erro relativo em relação aos valores desejados de apenas 3,01\%.

$F C_{1}, Y_{F 1}$ e $P R_{1}$ apresentam o mesmo erro para cada caso, porque, pela definição das Equações (1), (2) e (4), todas são em função da potência de saída do gerador dividida pela potência nominal.

Por fim, o estudo de caso usando AF foi feito com o intuito de verificar a melhora que seria obtida em relação aos TDNN A e B. Neste contexto, esta melhora foi de fato evidenciada, como pode ser vista no valor do erro que é correspondente a $1,22 \%$, comparados aos $5,14 \%$ e $7,11 \%$, respectivamente. A grande desvantagem da utilização deste tipo de rede neste estudo é a necessidade de uma boa aproximação dos dados de entrada, que são variáveis meteorológicas. Nos dias de hoje, ainda há bastantes dificuldades para se prever com boa precisão estes valores. Desta maneira, caso dados imprecisos sejam adicionados àquela rede, a resposta da mesma também será imprecisa. 


\section{CONCLUSÕES}

Os resultados mostraram que a aplicação de redes perceptron multicamadas do tipo TDNN e AF em problemas de previsão de geração de energia elétrica em SFCR é bastante promissora. Resultados satisfatórios foram encontrados para um SFCR instalado na USP, considerando que tais redes neurais são de simples implementação computacional.

Na aplicação de redes TDNN, verificou-se a importância de se ter um conhecimento prévio do dia a ser realizada a previsão: se será chuvoso, nublado ou ensolarado. Neste sentido, os resultados mostraram uma forte tendência para que melhores estimativas da rede neural possam ser alcançadas caso estas informações sejam apropriadamente definidas para o dia em que se deseja prever o perfil da geração. A aplicação da rede AF apresentou bons resultados, embora apresente a desvantagem de necessitar de dados de entrada com boa precisão para o dia em que se deseja realizar a previsão. Porém, salienta-se que o estudo proposto em aplicação em tempo real, por exemplo, no contexto de operação de microgrids, pode apresentar desvantagens, tais como a necessidade de treinamento de novas situações.

Em todo este estudo, como trabalhos futuros, é interessante incorporar a utilização das técnicas de validação cruzada para determinação do número de neurônios e entradas das redes; modificação do número de dias para conjunto de treinamento; realização de análises mais criteriosas quanto aos benefícios advindos da divisão em classes do tipo ensolarado, nublado e chuvoso; aplicar ANN para previsão de ventos e; determinação de uma heurística ou metaheurística para uma boa classificação das mesmas.

\section{AGRADECIMENTOS}

Os autores gostariam de agradecer à Universidade de São Paulo e ao suporte da Coordenação de Aperfeiçoamento de Pessoal de Nível Superior (CAPES) e da Fundação de Amparo à Pesquisa do Estado de São Paulo (FAPESP).

\section{REFERÊNCIAS}

Abdallah, H. H., Chtourou, M., Guesmi, T. and Quali, A. (2006). Feedforward neural network-based transient stability analysis of electric power systems, International Transactions on Electrical Energy Systems, 16 (6): pp. 577-590.

Betz, S. (2019). Geração Distribuída Solar Fotovoltaica: Panorama, Oportunidades e Desafios. p. 1 - 29.

Fatemi, S. A., Kuh, A. and Fripp, M. (2016), Online and batch methods for solar radiation forecast under asymmetric cost functions, Renewable Energy, 91: pp. 397-408, June 2016.

Filho, G. F. P., Vasconcelos, F. M., Macêdo, W. N., Manito, A. R. A. and Galhardo, M. A. B. (2010). Ferramenta Computacional para Análise e Projeto de Sistemas Fotovoltaicos Conectados à Rede Elétrica, III Congresso Brasileiro de Energia Solar (CBENS), Belém.

Flaticon (2017). Database of free icons. Disponível em: https://www.flaticon.com. Acesso em 20 Oct. 2017.
Gergaud, O., Multon, B. and Ahmed, H. B. (2002), Analysis and Experimental Validation of Various Photovoltaic System Models, $7^{\text {th }}$ International Eletrimacs Congress, Montreal, pp. 1 - 6, Canada.

Goedtel, A., Silva, I. N., Semi, P. J. A., Suetake, M., Nascimento, C. F. and Silva, S. A. O. (2013), Speed Estimation for Induction Motor Using Neural Networks Method, IEEE Latin America Transactions, 11 (2): pp. 768-778, https://doi.org/10.1109/TLA.2013.6533966.

Golestaneh, F., Pinson, P. and Gooi, H. B. (2016), Very Short-Term Nonparametric Probabilistic Forecasting of Renewable Energy Generation - With Application to Solar Energy, IEEE Transactions on Power Systems, 31 (5): pp. 3850-3863.

Júnior, H. F., Trigoso, F. B. M. and Cavalcanti, J. A. M. (2017). Review of distributed generation with photovoltaic grid connected systems in Brazil: Challenges and prospects, Renewable and Sustainable Energy Reviews 75: pp. 469-475.

Macêdo, W. N. (2006). Análise do Fator de Dimensionamento do Inversor Aplicado a Sistemas Fotovoltaicos Conectados à Rede. Tese de Doutorado, Programa Interunidades de Pós-Graduação em Energia (EP/FEA/IEE/IF). 201 p. USP, São Paulo.

Martín, E. C. (1998). Edificios Fotovoltaicos Conectados a la Red Elétrica: Caracterización y Análisis. Tese de Doutorado, Universidade Politécnica de Madri.

Mohammed, A. A., and Aung, Z. (2016). Ensemble Learning Approach for Probabilistic Forecasting of Solar Power Generation, Energies, 9 (12): pp. 1-17.

Oliveira, L. W. and Maria, T. C. J. (2017). Planning of Renewable Generation in Distribution Systems Considering Daily Operating Periods, IEEE Latin America Transactions, 15 (5): pp. 901-907.

Persson, C., Bacher, P., Shiga, T. and Madsen, H. (2017). Multi-site solar power forecasting using gradient boosted regression trees, Solar Energy, 150: pp. 423-436.

Raza, M. Q., Nadarajah, M. and Ekanayake, C. (2016). On recent advances in PV output power forecast, Solar Energy, 136: pp. 125-144.

Saraiva, F. O., Bernardes, W. M. S. and Asada, E. N. (2015), A framework for classification of non-linear loads in smart grids using Artificial Neural Networks and MultiAgent Systems, Neurocomputing, 170: pp. 328-338.

Silva, I. N., Spatti, D. H. and Flauzino, R. A. (2010), Redes neurais artificiais para engenharia e ciências aplicadas, Ed. Artliber Ltda., São Paulo.

Takeda, H. (2017). Short-term ensemble forecast for purchased photovoltaic generation, Solar Energy, 149: pp. 176-187.

Zilles, R., Macêdo, W. N., Galhardo, M. A. B. and Oliveira, S. H. F (2012). Sistemas fotovoltaicos conectados à rede elétrica. Oficina de Texto, São Paulo. 